\title{
Efficacy and outcome of platelet rich plasma in management of primary osteoarthritis Knee; A prospective study
}

\author{
Vivek Sharma ${ }^{1}$, Rashid Anjum ${ }^{2 *}$, Ravish ${ }^{3}$, Ravinder Singh ${ }^{4}$ \\ 1,2 Assistant Professor, ${ }^{3}$ Orthopaedic Surgeon, ${ }^{4}$ Professor, Dept. of Orthopaedic, ${ }^{1}$ GMC Kathua, Jammu Kashmir, ${ }^{2}$ ASCOMS and Hospital, \\ Jammu Kashmir, ${ }^{\mathbf{3}, \mathbf{4}}$ Maharishi Markandeshwar Institute of Medical Sciences and Research, Ambala, Haryana, India \\ *Corresponding Author: Rashid Anjum \\ Email: raashidanjum@gmail.com
}

\begin{abstract}
Introduction: The present management line of osteoarthritis of knee is mostly symptomatic, therefore the need to investigate treatment modality which addresses the disease process in itself. This study was undertaken with an aim to evaluate the efficacy of platelet rich plasma in primary osteoarthritis of knee.

Materials and Methods: This prospective study was done on 67 knees in 60 patients of early primary osteoarthritis of the knee. Three injections of platelet rich plasma were given at four weeks interval and the outcome was evaluated using WOMAC and VAS score at pre injection, 3 weeks, 12 weeks and 24 weeks. Ahlback's grading was done at pre-injection and at 6 months.

Results: Most of the cases in our series were in the age group of 51-60 years with female predominance (66.7\%), 46 cases had grade 2 disease and the remaining had grade 1 involvement. Improvement in WOMAC score parameters started at 3 weeks with progressive improvement at subsequent follow-ups. WOMAC score parameters (pain, stiffness, physical function and total score) at pre injection were $14.31,5.01,36.52$ and 55.75 and at 6 months follow up were 6.54, 2.03, 19.07 and 27.66 respectively. Similar improvement was seen in VAS score with pre injection score of 6.45 and final score of 3.76. The average WOMAC score was less for grade 1 as compared to grade 2 (45.52 and 60.41). Improvement was seen in $89.55 \%$ of cases though the level varied from good to mild improvement. There was no complication related to the treatment in our series.

Conclusion: The initial results conclude that PRP is an effective treatment modality in early grades of primary Osteoarthritis of knee. However, further high evidence studies are required to look for the long-term effects and to confirm the findings of this study.
\end{abstract}

Keywords: Platelet rich plasma, osteoarthritis, intra articular injection, PRP in knee.

\section{Introduction}

Osteoarthritis (OA) is the most predominant form of arthritis and the main cause of disability in the people aged 65 and older. ${ }^{1}$ It is a clinically varied and poorly understood disease. Although most research has looked at destruction of the cartilage, arthritis is often now considered in terms of organ failure. ${ }^{2}$ The available management strategies at present mostly provide symptomatic relief but do not in general, address the disease process itself. The role of growth factors and autologous platelet rich plasma (PRP) is recently being considered as a therapeutic possibility to augment healing of chondral injuries and modify the course of early degenerative arthritis. The concept that application of PRP would improve cartilage repair is based on the physiological role of platelets in wound healing. There are over 1500 proteins within platelets including the growth factors like PDGF, VEGF, TGF-beta, FGF, and EGF that are known to play a pivotal role in the normal healing process. $^{3}$

The apposite treatment for cartilage injury remains a clinical challenge despite advances in surgical procedures and techniques. Even after promising preclinical findings and huge interest in its clinical application most questions on PRP still remains unanswered.

\section{Materials and Methods}

This prospective study was conducted in a tertiary care centre of northern India on 60 patients of primary early OA diagnosed by American College of Rheumatology Criteria ${ }^{4}$ after obtaining an informed written consent and staged as per Ahlback's radiological grading5. Bilateral knee involvement was seen in seven patients making the final figure of 67 knees. The patients with Grade 1 and Grade 2 disease, aged 35-70 years and no history of any intraarticular injection in previous 3 months were included in the study. The platelet rich plasma was prepared from $100 \mathrm{ml}$ patient's venous blood collected in $100 \mathrm{ml}$ bag with CPD-A (Citrate Phosphate Dextrose and Adenine) as anticoagulant. The blood was transferred into two $50 \mathrm{ml}$ tubes which were then centrifuged for 15 minutes at $1500 \mathrm{rpm}$ on a table top centrifuge. The blood was separated into platelet rich plasma (PRP) and residual RBC's with the buffy coat. The PRP was extracted through a pipette and transferred to a test tube. It was then supplied in a syringe in a quantity of $8 \mathrm{ml}$ meant for injection into one knee. The platelet concentrate was injected by lateral approach by 18-20 Gauge needle. Patients were given a total of three injections at four weeks interval. Pre-injection and post injection evaluation at three weeks, three months and six months was done using VAS ${ }^{6}$ and WOMAC ${ }^{7}$ score. Ahlback's grading wherever possible was done at six months.

\section{Results}

There were total 60 patients, of which $40(66.7 \%)$ were females and $20(33.3 \%)$ were males. Majority of the patients were in the age group of 51-60 years constituting 41.7\%. 46 knees revealed grade 2 disease whereas grade 1 disease was seen in 21 knees. The age and sex distribution of patients is given in the table 1 . 
Table 1: Age and sex distribution of patients.

\begin{tabular}{|c|c|c|c|}
\hline Age (in years) & Females & Males & Total \\
\hline$<40$ & 6 & & $6(10.0 \%)$ \\
\hline $41-50$ & 12 & 5 & $17(28.3 \%)$ \\
\hline $51-60$ & 15 & 10 & $25(41.7 \%)$ \\
\hline $61-70$ & 7 & 5 & $12(20.0)$ \\
\hline Total & 40 & 20 & $60(100 \%)$ \\
\hline
\end{tabular}

Individual mean WOMAC score and total mean WOMAC score were analyzed. Improvement in symptoms started showing up at 3 weeks which gradually improved over subsequent follow ups as depicted by $\mathrm{p}$ value of 0.000 and decrease in the mean values as shown in table 2.

VAS score decreased from 6.45 at pre injection to 6.22 , 4.54 , and 3.76 at subsequent follow ups till 6 months. The improvement seen was statistically significant ( $\mathrm{p}$ value 0.000). (Table 3).

The mean Total WOMAC score based on Ahlback's grading done at pre injection and third follow up was calculated and it was observed that mean Total WOMAC score was less for grade 1 in comparison to grade 2 osteoarthritis. Twenty-one patients had mild pain and 2 patients had moderate pain which subsided in 2-3 days without any intervention. One patient experienced episode of sweating and tachycardia. No serious complication was encountered. Improvement as per patient version was asked at the end of six months. Sixty patients comprising $89.55 \%$ showed improvement though the level varied from mild to good improvement. (Table 4).

\section{Discussion}

In the current literature only a handful studies have studied the use of PRP in primary osteoarthritis of knee. However, there is lack of standardization among the available data. As a result, the evidence to support the clinical use of PRP as a treatment modality is uncertain in primary osteoarthritis of knee. The mean age of patients in our study was 53.75 with a range of 35-70 years. Similar results were observed by Filardo et $\mathrm{al}^{8}$ and Patel et al, ${ }^{9}$ whereas, in a study by Sanchez et $\mathrm{al}^{10}$ the mean age of the patients was 63.53 years which was because of inclusion of advanced cases of osteoarthritis in their study.

We noted a female predominance in our study which is in agreement with the other studies. ${ }^{7,8}$ However, in contrast, Kon et $\mathrm{al}^{11}$ and Sampson et $\mathrm{al}^{12}$ observed male preponderance in their study.

We experienced a definite correlation with Ahlback's grading. Total WOMAC score was less for grade 1 in contrast to grade 2 at baseline as well as at third follow up. Kon et $\mathrm{al}^{11}$ concluded that younger patients with lower degree of degenerative chondropathy attained better results in comparison to patients affected by early osteoarthritis, which in turn showed higher improvement in comparison to patients of advanced osteoarthritis. Patel et $\mathrm{al}^{9}$ also obtained a similar correlation with Ahlback's grading in both A and $\mathrm{B}$, with grade 1 knees having lower mean pain and other WOMAC scores than grade 2 knees $(\mathrm{P}=0.016$ for group $\mathrm{A}$, $\mathrm{P}=0.005$ for group $\mathrm{B}$ ).

Pain being the most troublesome symptom, WOMAC and VAS score assessment revealed alike trends. Pain decreased significantly at first follow up which was comparable to the other published literature. However, the analysis of published data showed worsening at final 6 months follow up, which raises a question if the effect of PRP is provisional or it has the ability to modify the disease process or serial injections are required for long term relief? This is still an unanswered question and future research work is required to prove the same.

Sanchez et $\mathrm{al}^{10}$ in his study observed $33.3 \%$ success rate at week 5 , Kon et al $^{11}$ reported $80 \%$ satisfied patients and Patel et $\mathrm{al}^{9}$ had $67.3 \%$ satisfied patients in group A and $64 \%$ satisfied patients in group B at 6 month follow-up, in comparison with the control group in which only $4.3 \%$ were satisfied. We reported $89.55 \%$ improvement though the level varied from good improvement to mild improvement.

None of the previous studies in literature has mentioned any serious side effects on administration of platelet rich plasma. Same results were validated in our study. The main complication was mild pain which was probably due to an interventional procedure and natural response of body to inflammatory mediators.

We assume that platelet rich plasma possibly has a role in the treatment of osteoarthritis which is supported by the improvement seen in VAS and WOMAC scores in our study at short term follow up. This improvement in our study is possibly due to the anti-inflammatory properties or due to release of various growth factors and not because of chondral remodeling. If chondral remodeling was the cause for the improvement of symptoms, the benefit would have started later and lasted for a longer duration, however to comment on the same in our study, longer follow up is needed. Furthermore, high value studies with longer follow up are required to evaluate, if the effect of PRP is temporary or it has the ability to modify the disease process.

Limitations in our study were time period and the evaluation parameters used which were WOMAC and VAS score only because of the fact that pain is the main presenting symptom. Another drawback was lack of evidence if there is any cartilage regeneration as there was no follow up investigations other than X-ray which is not a sensitive device to document the change if there is any.

Table 2: WOMAC score (mean) at pre-injection, 3 weeks, 3 months and 6 months interval

\begin{tabular}{|l|c|c|c|c|}
\hline \multicolumn{1}{|c|}{ Parameters } & Pre injection & First follow up & Second follow up & Third follow up \\
\hline Pain & $14.31 \pm 2.12$ & $13.03 \pm 2.47$ & $8.99 \pm 2.23$ & $6.54 \pm 3.19$ \\
\hline Stiffness & $5.01 \pm 1.34$ & $4-31 \pm 1.51$ & $3.01 \pm 1.39$ & $2.03 \pm 1.51$ \\
\hline Physical function & $36.52 \pm 8.79$ & $35.03 \pm 9.06$ & $23.82 \pm 9.92$ & $19.07 \pm 8.46$ \\
\hline Total & $55.75 \pm 10.90$ & $52.34 \pm 11.54$ & $35.82 \pm 9.92$ & $27.66 \pm 12.48$ \\
\hline
\end{tabular}


Table 3: VAS score means with time

\begin{tabular}{|c|c|}
\hline VAS score & Mean \pm SD \\
\hline Pre injection & $6.45 \pm 1.22$ \\
\hline $1^{\text {st }}$ follow up & $6.22 \pm 1.19$ \\
\hline $2^{\text {nd }}$ follow up & $4.54 \pm 1.11$ \\
\hline $3^{\text {rd }}$ follow up & $3.76 \pm 1.37$ \\
\hline
\end{tabular}

Table 4: Status of improvement in patients at six months

\begin{tabular}{|c|c|}
\hline Number of knees (N=67) & Satisfaction \\
\hline $17(25.4 \%)$ & Good improvement \\
\hline $23(34.3 \%)$ & Moderate improvement \\
\hline $20(29.9 \%)$ & Mild improvement \\
\hline $07(10.4 \%)$ & No improvement \\
\hline- & Worsened \\
\hline
\end{tabular}

\section{Conclusion}

We conclude that PRP appears to be a good treatment option for early grades of primary osteoarthritis of knee at short term follow up. However, more high value evidence based studies are required to prove its effectiveness on longer follow up and in late stages of OA cases.

\section{Conflict of Interest: None.}

\section{References}

1. Garstand SV, Stitik TP. Osteoarthiritis: epidemiology, risk factors, and pathophysiology. Am J Phys Med Rehabil 2006;85(Suppl) osteoarthritis:2-11.

2. Cianflocco AJ. Pathophysiolgy and diagnosis of osteoarthritis of the knee. J Fam Prac 2011;60:S37-40.

3. Lauder SN, Carty SM, Carpenter CE, Hill RJ, Talamas F, Bondeson J, et al. interleukin-1 beta induced activation of nuclear factor-kappab can be inhibited by novel pharmacological agents in osteoarthritis. Rheumatol (Oxford) 2007;46(5):752-8.

4. Altman R, Asch E. Bloch D, Bole G, Borenstein D, Brandt K, et al. development of criteria for the classification and reporting of osteoarthritis. Classification of osteoarthritis of the knee. Arthritis Rheum 1986;29:1039-49.

5. Petersson IF, Boegard T, Silman AJ, Saxne T, Svensson B. Radiographic osteoarthritis of the knee classified by the Ahlback and Kellgren \& Lawrence systems for the tibiofemoral joint in people aged 35-54 years with chronic knee pain. Ann rheum Dis 1997;56:493-6.

6. Hawker GA, Mian S, Kendzerska T, French M. measures of adult pain. Arthritis Care Res 2011;63(S11):S240-52.

7. Bellamy N, Wilson C, Hendrikz J, Whitehouse SL, Patel B, Dennison S, et al. EDC Study Group. J Clin Epidemiol 2011;64(2):182-90.

8. Filardo G, Kon E, Buda R, Timoncini A, Di M artino A, Cenacchi A, et al. Platelet-rich plasma intra-articular knee injections for the treatment of degenerative cartilage lesions and osteoarthritis. Knee Surg Sports Traumatol Arthrosc 2011;19(4):528-35.

9. Patel S, Dhillon MS, Aggarwal S, Marwaha N, Jain A. Treatment with platelet -rich plasma is more effective than placebo for knee osteoarthritis: a prospective double-blind, randomized trial. Am J Sports Med 2013;41(2):356-64.

10. Sanchez M, Anitua E, A Zofra J, Aguirre JJ, Andia I. intraarticular injection of an autologous preparation rich in growth factors for the treatment of knee OA: a retrospective cohort study. Clin Exp Rheumatol 2008;26(5):910-3.
11. Kon E, Buda R, Filardo G, Di Martino A, Timoncini A, Cenachhi A, et al. platelet-rich plasma: intra-articular knee injections produced favourable results on degenerative cartilage lesions. Knee Surg Sports Traumatol Arthrosc 2010;18(4):472-9.

12. Sampson S, Reed M, Silvers H, Meng M, Mandelbaum B. Injection of platelet-rich plasma in patients with primary and secondary knee osteoarthritis: a pilot study. Am J Phys Med Rehabil 2010;89(12):961-9.

How to cite this article: Sharma V, Anjum R, Ravish, Singh R. Efficacy and outcome of platelet rich plasma in management of primary osteoarthritis Knee; A prospective study. Indian J Orthop Surg 2019;5(2):1113. 